JOURNAL OF

FUNCTION SPACES AND APPLICATIONS

Volume 2, Number 1 (2004), 55-69 (c) 2004, Scientific Horizon http://www.jfsa.net

\title{
A trace inequality for generalized potentials in Lebesgue spaces with variable exponent
}

\author{
David E. Edmunds, Vakhtang Kokilashvili and Alexander Meskhi
}

(Communicated by Vladimir Stepanov)

1991 Mathematics Subject Classification. 31C15, 42B35, $47 \mathrm{~B} 34$.

Keywords and phrases. Trace inequality, Riesz potentials, generalized Lebesgue spaces, spaces of homogeneous type.

\footnotetext{
Abstract. A trace inequality for the generalized Riesz potentials $I^{\alpha(x)}$ is established in spaces $L^{p(x)}$ defined on spaces of homogeneous type. The results are new even in the case of Euclidean spaces. As a corollary a criterion for a two-weighted inequality in classical Lebesgue spaces for potentials $I^{\alpha(x)}$ defined on fractal sets is derived.
}

\section{Introduction}

In this paper several extensions of D. Adams's trace inequality [1] (see also [2]) are established. We prove a trace inequality for generalized Riesz potentials in Lebesgue spaces with variable exponent. This result can be interpreted as an inequality of Sobolev type. At the same time we derive criteria for the validity of a two-weighted inequality in the case of classical Lebesgue spaces when the exponent involved in the definition of the potential is variable. Lebesgue spaces $L^{p(x)}$ with variable exponent and operators in these spaces are intensively studied nowadays. The considerable interest in such spaces in recent years has been stimulated by applications to models with the so-called non-standard local growth in fluid 
mechanics, elasticity theory, in variational calculus, differential operators; see for example [22], [16], [5] and references therein.

Let

$$
I^{\alpha} f(x)=\int_{R^{n}} \frac{f(y)}{|x-y|^{n-\alpha}} d y, \quad 0<\alpha<n,
$$

be the Riesz potential. A theorem of Sobolev for $I^{\alpha}$ in Lebesgue spaces with variable exponent was proved quite recently [4] (see also [17], [8], [12-13]).

For Lebesgue spaces with constant exponent D. Adams [1] proved the following

Theorem A. Let $1<\gamma<\lambda<\infty$ and $0<\alpha<n / \gamma$, let $\rho$ be a measurable function on $R^{n}$, positive a.e.. Then the trace inequality

$$
\left(\int_{R^{n}}\left(\left|I^{\alpha} f(x)\right| \rho(x)\right)^{\lambda} d x\right)^{1 / \lambda} \leq c\left(\int_{R^{n}}|f(x)|^{\gamma} d x\right)^{1 / \gamma}
$$

where $f \in L^{\gamma}\left(R^{n}\right)$, holds if and only if

$$
\sup _{\substack{x \in R^{n} \\ r>0}} r^{\lambda(\alpha-n / \gamma)} \int_{B(x, r)} \rho^{\lambda}(x) d x<\infty,
$$

where $B(x, r)$ is the open ball in $R^{n}$ with center $x$ and radius $r$.

In [6] (see Chapter 6) the reader can find several extensions of Theorem A for spaces of homogeneous type and also for the so-called nonhomogeneous spaces. These results concern the classical Lebesgue spaces.

We aim to generalize Theorem A in several directions. To explain this we need some definitions.

Let $(X, d, \mu)$ be a space of homogeneous type $(S H T)$, that is, a topological space endowed with a complete measure $\mu$ such that the space of compactly supported continuous functions is dense in $L^{1}(X, d \mu)$ and there exists a non-negative real-valued function $d: X \times X \rightarrow R$ satisfying

(i) $d(x, x)=0$ for all $x \in X$;

(ii) $d(x, y)>0$ for all $x \neq y, x, y \in X$;

(iii) there is a constant $a_{0}$ such that $d(x, y) \leq a_{0} d(y, x)$ for all $x, y, z \in X$;

(iv) there is a constant $a_{1}$ such that

$$
d(x, y) \leq a_{1}(d(x, z)+d(z, y))
$$

for all $x, y, z \in X$;

(v) for every neighbourhood $V$ of $x$ there is $r>0$ such that the ball $B(x, r)=\{y \in X: d(x, y)<r\}$ is contained in $V$;

(vi) there is a constant $b$ such that $\mu B(x, 2 r) \leq b \mu B(x, r)<\infty$ for every $x \in X$ and all $r, 0<r<\infty$. 
For the definition, some properties and examples of $S H T$ see e.g. [10].

We now give the definition of Lebesgue spaces with variable exponent (see [11], [14], [18], [7], [9]).

Let $p(x)$ be a $\mu$-measurable function on $X$ such that

$$
1<p_{-} \leq p(x) \leq p_{+}<\infty, \quad x \in X
$$

where

$$
p_{-}:=\underset{x \in X}{\operatorname{essinf}} p(x), \quad p_{+}:=\underset{x \in X}{\operatorname{ess} \sup } p(x) .
$$

By $L^{p(x)}$ we denote the space of all functions $f$ on $X$ such that

$$
A_{p}(f):=\int_{X}|f(x)|^{p(x)} d \mu(x)<\infty .
$$

This is a Banach function space with respect to the norm

$$
\|f\|_{L^{p(\cdot)}}:=\inf \left\{\lambda>0: A_{p}(f / \lambda) \leq 1\right\} .
$$

Let $w$ be a $\mu$ - measurable almost everywhere positive function on $X$. Such functions are called weights.

The generalized weighted Lebesgue space $L_{w}^{p(\cdot)}$ is defined to be the set of all measurable functions $f$ for which

$$
\|f\|_{L_{w}^{p(\cdot)}}:=\|f w\|_{L^{p(\cdot)}}<\infty .
$$

Note that $L_{w}^{p(\cdot)}$ is a Banach space (see e.g. [7]). When $p(x) \equiv p=$ const, then $L_{w}^{p(\cdot)}(X)$ coincides with the weighted Lebesgue space $L_{w}^{p}(X)$.

If $w$ is a positive and locally integrable function on $X$, then for any $\mu^{-}$ measurable set $e$ we put

$$
w e=\int_{e} w(x) d \mu
$$

\section{Statements of Main Results}

In the sequel $X$ will be assumed to satisfy the condition

$$
B(x, R) \backslash B(x, r) \neq \emptyset
$$

for all $r$ and $R, 0<r<R \leq c$, where $c$ is some positive constant. We shall assume also that $\mu X<\infty$ and there exist positive constants $c_{0}$ and $s$ such that

$$
\mu B(x, r) \leq c_{0} r^{s}
$$


for all $x \in X$ and all $r>0$. By $l$ we denote $\operatorname{diam} X:=\sup \{d(x, y): x, y \in$ $X\}$.

Definition 1. Let $g$ be a continuous function on $X$. We say that $g$ belongs to $W-\operatorname{Lip}(X)$ (weak Lipschitz condition) if there exists a positive constant $c$ such that

$$
|g(x)-g(y)| \leq \frac{c}{-\ln (d(x, y))}
$$

for all $x, y \in X$ with $0<d(x, y) \leq 1 / 2$. The main object of our study is the generalized Riesz potential

$$
I^{\alpha(x)} f(x)=\int_{X}(d(x, y))^{\alpha(x)-s} f(y) d \mu(y), \quad 0<\alpha(x)<s .
$$

Our main goal is to prove two statements. The first concerns criteria for a two-weight inequality for the operator $I^{\alpha(x)}$ in a weighted Lebesgue space with constant exponent. A trace inequality for $I^{\alpha(x)}$ in Lebesgue spaces with variable exponent will be given in Theorem II. Both statements are new even in case of Euclidean space. Then we derive some corollaries and indicate the interesting special cases, such as potentials on thin sets.

Theorem I. Let $1<\gamma<\lambda<\infty, 0<\alpha(x)<s$ and $\alpha \in W-\operatorname{Lip}(X)$; let $\rho$ and $w$ be weights.

Then the operator $I^{\alpha(x)}$ is bounded from $L_{w}^{\gamma}(X)$ to $L_{\rho}^{\lambda}(X)$ if and only if

$$
\begin{aligned}
& \sup _{\substack{x \in X \\
0<r<l}}\left(\rho^{\lambda} B(x, N r)\right)^{1 / \lambda} \\
& \quad \times\left(\int_{X \backslash B(x, r)} w^{-\gamma^{\prime}}(y)(d(x, y))^{(\alpha(x)-s) \gamma^{\prime}} d \mu(y)\right)^{1 / \gamma^{\prime}}<\infty
\end{aligned}
$$

and

$$
\begin{aligned}
& \sup _{\substack{x \in X X \\
0<r<l}}\left(w^{-\gamma^{\prime}} B(x, N r)\right)^{1 / \gamma^{\prime}} \\
& \quad \times\left(\int_{X \backslash B(x, r)} \rho^{\lambda}(y)(d(x, y))^{(\alpha(x)-s) \lambda} d \mu(y)\right)^{1 / \lambda}<\infty
\end{aligned}
$$

where $N=2 a_{1}\left(1+2 a_{0}\right)$. The constants $a_{0}$ and $a_{1}$ are from the definition of $S H T$.

Corollary 1. Let $1<\gamma<\lambda<\infty, \alpha \in W-\operatorname{Lip}(X)$ and $\sup _{x \in X} \alpha(x)<s / \gamma$. Then 
(i) the operator $I^{\alpha(x)}$ acts boundedly from $L^{\gamma}(X)$ into $L_{\rho}^{\lambda}(X)$ if

$$
\sup _{\substack{x \in X \\ 0<r<l}} r^{\lambda(\alpha(x)-s / \gamma)} \int_{B(x, r)} \rho^{\lambda}(y) d \mu(y)<\infty .
$$

(ii) If

$$
b_{1} r^{s} \leq \mu B(x, r) \leq b_{2} r^{s}
$$

for some positive constants $b_{1}$ and $b_{2}$, then the condition (2.4) is also necessary for the boundedness of $I^{\alpha(x)}$ from $L^{\gamma}(X)$ to $L_{\rho}^{\lambda}(X)$.

Theorem II. Let $p(\cdot)$ and $q(\cdot)$ be measurable functions on $X$ with $1<p_{-}<q_{+}<\infty, \alpha \in W-\operatorname{Lip}(X)$ and $\sup \alpha(x)<s / p_{-}$. Suppose that $v$ is a weight.

Then the condition

$$
\sup _{\substack{x \in X \\ 0<r<l}} r^{q+\left(\alpha(x)-s / p_{-}\right)} \int_{B(x, r)}(v(y))^{q(y)}(y) d \mu(y)<\infty
$$

implies the boundedness of $I^{\alpha(x)}$ from $L^{p(\cdot)}(X)$ to $L_{v}^{q(\cdot)}(X)$.

From this theorem for $I^{\alpha(x)}$ follows a statement of Sobolov type.

Corollary 2. Let $p(\cdot)$ and $q(\cdot)$ be arbitrary measurable functions on $X$ such that $1<p_{-}<q_{+}<\infty$. Suppose that $\alpha \in W-\operatorname{Lip}(X)$ and $s\left(\frac{1}{p_{-}}-\frac{1}{q_{+}}\right) \leq \inf _{x \in X} \alpha(x) \leq \sup _{x \in X} \alpha(x)<\frac{s}{p_{-}}$. Then $I^{\alpha(x)}$ acts boundedly from $L^{p(\cdot)}(X)$ into $L^{q(\cdot)}(X)$.

\section{Proofs of Main Results}

Let $k$ be a positive measurable function on $X \times X$ and let

$$
\mathcal{K} f(x)=\int_{X} k(x, y) f(y) d \mu(y)
$$

and

where $k^{*}(x, y)=k(y, x)$.

$$
\mathcal{K}^{*} f(x)=\int_{X} k^{*}(x, y) f(y) d \mu(y),
$$


Definition 3.1. We say that $k$ belongs to the class $V(k \in V)$ if there exists a positive constant $c$ such that

$$
k(x, y) \leq c k\left(x^{\prime}, y\right)
$$

for all $x, y$ and $x^{\prime}$ such that $d\left(x, x^{\prime}\right) \leq N d(x, y)$, where $N=2 a_{1}\left(1+2 a_{0}\right)$.

The following two-weighted criterion for the operator $K$ is known for classical Lebesgue spaces (see e.g. [10], Theorem 3.4.2 and [19]).

Theorem B. Let $1<\gamma<\lambda<\infty$ and let $k, k^{*} \in V$. Then the operator $K$ is bounded from $L_{w}^{\gamma}(X)$ to $L_{v}^{\lambda}(X)$ if and only if

$$
\sup _{\substack{x \in X \\ 0<r<l}}\left(v^{\lambda} B(x, N r)\right)^{1 / \lambda}\left(\int_{X \backslash B(x, r)} k^{\gamma^{\prime}}(x, y) w^{-\gamma^{\prime}}(y) d \mu(y)\right)^{1 / \gamma^{\prime}}<\infty
$$

and

$$
\sup _{\substack{x \in X \\ 0<r<l}}\left(w^{-\gamma^{\prime}} B(x, N r)\right)^{1 / \gamma}\left(\int_{X \backslash B(x, r)} k^{\lambda}(y, x) v^{\gamma}(y) d \mu(y)\right)^{1 / \lambda}<\infty .
$$

Note that the condition (2.1) is not needed for this result. Observe also that Theorem A is a consequence of Theorem B.

Now we shall derive Theorem I from Theorem B.

Proposition 3.1. Let $0<\alpha(x)<s$ for all $x \in X$ and $\alpha \in W-\operatorname{Lip}(X)$. Then there exist positive constants $c_{1}$ and $c_{2}$ such that for all $x, y \in X$

$$
c_{1}(d(x, y))^{\alpha(y)-s} \leq(d(x, y))^{\alpha(x)-s} \leq c_{2}(d(x, y))^{\alpha(y)-s} .
$$

Proof. To prove the right-side of inequality (3.3) let $d(x, y) \leq \frac{1}{2}$. We have

$$
(d(x, y))^{\alpha(x)-s}=(d(x, y))^{\alpha(x)-\alpha(y)+\alpha(y)-s} .
$$

Further,

$$
\begin{aligned}
\left|\ln (d(x, y))^{\alpha(x)-\alpha(y)}\right| & =|\alpha(x)-\alpha(y)||\ln d(x, y)| \\
& \leq \frac{c}{-\ln d(x, y)}(-\ln d(x, y)) \\
& =c .
\end{aligned}
$$


Hence

$$
e^{-c} \leq(d(x, y))^{\alpha(x)-\alpha(y)} \leq e^{c}
$$

From the latter inequality and (3.4) we obtain the right-hand side of inequality (3.3). Similarly the left-hand side of (3.3) follows. If $1 / 2 \leq$ $d(x, y) \leq l$ then, $(3.3)$ is obvious.

Proof of Theorem I. Suppose that $k(x, y)=(d(x, y))^{\alpha(x)-s}$. Then we shall prove that $k$ and $k^{*}$ belong to the class $V$. Let

$$
d\left(x, x^{\prime}\right) \leq N d(x, y)
$$

We have

$$
\begin{aligned}
d\left(x^{\prime}, y\right) & \leq a_{1}\left(d\left(x^{\prime}, x\right)+d(x, y)\right) \\
& \leq a_{1}\left(a_{0} N d(x, y)+d(x, y)\right) \\
& =a_{1}\left(a_{0} N+1\right) d(x, y)
\end{aligned}
$$

From the last inequality and Proposition 3.1 we conclude that

$$
\begin{aligned}
k(x, y) & \leq c_{1}(d(x, y))^{\alpha(y)-s} \\
& \leq c_{2}\left(d\left(x^{\prime}, y\right)\right)^{\alpha(y)-s} \\
& \leq c_{3}\left(d\left(x^{\prime}, y\right)\right)^{\alpha\left(x^{\prime}\right)-s} \\
& =c_{3} k\left(x^{\prime}, y\right) .
\end{aligned}
$$

The inclusion $k^{*} \in V$ follows analogously. Now applying Theorem B we come to the desired result.

Proof of Corollary 1. To prove (i) it suffices to show that the condition (2.4) implies conditions (2.2) and (2.3).

Denote

$$
D_{k}(x, r)=B\left(x, 2^{k+1} r\right) \backslash B\left(x, 2^{k} r\right), \quad k=0,1,2, \ldots
$$

We have

$$
\begin{aligned}
& \left(\rho^{\lambda} B(x, N r)\right)^{\gamma^{\prime} / \lambda} \int_{X \backslash B(x, r)}(d(x, y))^{(\alpha(x)-s) \gamma^{\prime}} d \mu(y) \\
& \quad=\left(\rho^{\lambda} B(x, N r)\right)^{\gamma^{\prime} / \lambda} \sum_{k=0}^{\infty} \int_{D_{k}(x, r)}(d(x, y))^{(\alpha(x)-s) \gamma^{\prime}} d \mu(y)
\end{aligned}
$$




$$
\begin{aligned}
& \leq\left(\rho^{\lambda} B(x, N r)\right)^{\gamma^{\prime} / \lambda} \sum_{k=0}^{\infty} \mu D_{k}(x, r)\left(2^{k} r\right)^{(\alpha(x)-s) \gamma^{\prime}} \\
& \leq c\left(\rho^{\lambda} B(x, N r)\right)^{\gamma^{\prime} / \lambda} \sum_{k=0}^{\infty}\left(2^{k} r\right)^{(\alpha(x)-s) \gamma^{\prime}+s} \\
& \leq c\left(\rho^{\lambda} B(x, N r)\right)^{\gamma^{\prime} / \lambda} r^{(\alpha(x)-s) \gamma^{\prime}+s} \sum_{k=0}^{\infty} 2^{k\left((\alpha(x)-s) \gamma^{\prime}+s\right)} \\
& \leq c .
\end{aligned}
$$

In the last inequality we used (2.4) and the condition $\sup _{x \in X} \alpha(x)<\frac{s}{\gamma}$. Further

$$
\begin{aligned}
r^{s \lambda / \gamma^{\prime}} & \int_{X \backslash B(x, r)} \rho^{\lambda}(y)(d(x, y))^{(\alpha(x)-s) \lambda} d \mu(y) \\
= & r^{\frac{s \lambda}{\gamma^{\prime}}} \sum_{k=0}^{\infty} \int_{D_{k}(x, r)} \rho^{\lambda}(y)(d(x, y))^{(\alpha(x)-s) \lambda} d \mu(y) \\
& \leq r^{\frac{s \lambda}{\gamma^{\prime}}} \sum_{k=0}^{\infty}\left(2^{k} r\right)^{(\alpha(x)-s) \lambda} \int_{D_{k}(x, r)} \rho^{\lambda}(y) d \mu(y) \\
& =\sum_{k=0}^{\infty}\left(2^{k} r\right)^{(\alpha(x)-s) \lambda+\frac{\lambda s}{\gamma^{\prime}}} 2^{-k \frac{\lambda s}{\gamma^{\prime}}} \int_{D_{k}(x, r)} \rho^{\lambda}(y) d \mu(y) \\
& \leq c_{5} \sum_{k=0}^{\infty} 2^{-k \frac{\lambda s}{\gamma^{\prime}}} \\
& <\infty .
\end{aligned}
$$

Now we prove (ii). Let the operator $I^{\alpha(x)}$ be bounded from $L^{\gamma}(X)$ to $L_{\rho}^{\lambda}(X)$. By Theorem I it follows that condition (2.2) is satisfied. Let us show that in our case it means that (2.4) is valid. It is easy to see that the condition (2.5) implies the so-called reverse doubling condition, i.e. there exist some constants $\eta_{1}>1$ and $\eta_{2}>1$ such that

$$
\mu B\left(x, \eta_{1} r\right) \geq \eta_{2} \mu B(x, r)
$$

for small $r$ (For the proof of this fact for measures satisfying the doubling condition see e.g. [20], p. 11). 
Using reverse doubling condition and (2.5) from (2.2) we obtain

$$
\begin{gathered}
\left(\rho^{\lambda} B(x, r)\right)^{1 / \lambda} r^{\alpha(x)-s / \gamma} \leq \\
\leq\left(\rho^{\lambda} B(x, N r)\right)^{1 / \lambda}\left(\eta_{1} r\right)^{\alpha(x)-s}\left(\mu B\left(x, \eta_{1} r\right) / \mu B(x, r)\right)^{1 / \gamma^{\prime}}<\infty .
\end{gathered}
$$

Note that in this case we do not need the assumption $\sup \alpha(x)<s / \gamma$. For this it is enough that inf $\alpha(x)<s$.

Thus we proved that by (2.2), (2.4) is satisfied.

Let $\Omega$ be a bounded open set in $R^{n}$, and let $\mu$ be Lebesgue measure on $\Omega$. Then from Corollary 1 we conclude that the following statement is valid.

Proposition 3.2. Let $1<\gamma<\lambda<\infty$ and let $\alpha \in W-\operatorname{Lip}(\Omega)$ be such that

$$
\sup _{x \in \Omega} \alpha(x)<\frac{n}{\gamma}
$$

Then the operator $I^{\alpha(x)}$ acts boundedly from $L^{\gamma}(\Omega)$ into $L_{\rho}^{\lambda}(\Omega)$ if and only if

$$
\sup _{\substack{x \in \Omega \\ 0<r<l}} r^{\lambda\left(\alpha(x)-\frac{n}{\gamma}\right)} \int_{B(x, r)} \rho^{\lambda}(y) d y<\infty
$$

Proof of Theorem II. Let us recall that

$$
q_{-}:=\operatorname{ess}_{x \in X} \inf _{(x)} \text { and } q_{+}:=\underset{x \in X}{\operatorname{ess} \sup } q(x)
$$

Let $f \geq 0$ and suppose that

$$
\int_{X}(f(x))^{p(x)} d x \leq 1
$$

Then we have

$$
\begin{aligned}
& \int_{X}(v(x))^{q(x)}\left(\int_{X}(d(x, y))^{\alpha(x)-s} f(y) d \mu(y)\right)^{q(x)} d \mu(x) \\
& =\int_{X \cap\left\{x: I^{\alpha(x)} f(x) \geq 1\right\}}(v(x))^{q(x)}\left(I^{\alpha(x)} f(x)\right)^{q(x)} d x \\
& \quad+\int_{X \cap\left\{I^{\alpha(x)} f(x)<1\right\}}(v(x))^{q(x)}\left(I^{\alpha(x)} f(x)\right)^{q(x)} d x \equiv J_{1}+J_{2} .
\end{aligned}
$$

If we put $(\rho(x))^{\lambda}=(v(x))^{q(x)}$ in Corollary 1, then we obtain

$$
J_{1} \leq \int_{X \cap\left\{x: I^{\alpha(x)} f(x) \geq 1\right\}}(v(x))^{q(x)}\left(I^{\alpha(x)} f(x)\right)^{q+} d \mu(x)
$$




$$
\leq c\left(\int_{X}(f(x))^{p_{-}} d \mu(x)\right)^{q_{+} / p_{-}} .
$$

On the other hand, from condition (2.6) it is obvious that

$$
\sup _{\substack{x \in X, 0<r<l}} r^{q_{-}\left(\alpha(x)-s / p_{-}\right)} \int_{B(x, r)}(v(y))^{q(y)} d y<\infty .
$$

Therefore using Corollary 1 again we obtain

$$
J_{2} \leq \int_{X}(v(x))^{q(x)}\left(I^{\alpha(x)} f(x)\right)^{q_{-}} d \mu(x) \leq c\left(\int_{X}(f(x))^{p_{-}} d \mu(x)\right)^{q_{-} / p_{-}} .
$$

Now we observe that

$$
\begin{aligned}
\int_{X}(f(x))^{p_{-}} d \mu(x) & =\int_{X \cap\{f<1\}}(f(x))^{p_{-}} d \mu(x)+\int_{X \cap\{f \geq 1\}}(f(x))^{p_{-}} d \mu(x) \\
& \leq \int_{X}(f(x))^{p(x)} d \mu(x)+\mu(X) \\
& \leq 1+\mu(X) .
\end{aligned}
$$

Thus

$$
\int_{X}(v(x))^{q(x)}\left(I^{\alpha(x)} f(x)\right)^{q(x)} d \mu(x) \leq c .
$$

This proves the boundedness of $I^{\alpha(x)}$ from $L^{p(x)}(X)$ to $L_{v}^{q(x)}(X)$.

Proof of Corollary 2. It is clear that the condition (2.6) when $v(x) \equiv 1$ is satisfied if

$$
\inf _{x \in X} \alpha(x) \geq s\left(1 / p_{-}-1 / q_{+}\right) .
$$

From the previous results we can obtain the embedding theorem of Sobolev type for weighted spaces with variable exponent.

As above, let $\Omega$ be a bounded open set in $R^{n}$ and let $D^{k} u$ be the vector of all weak derivatives of $u$ of order $k$.

Proposition 3.3. Let $n \geq 2,1<p_{-}<q_{+}<\infty$ and let $k$ be any positive integer smaller than $n / p_{-}$. Suppose that the functions $p$ and $q$ satisfy the condition of Theorem II. If

$$
\sup _{\substack{x \in \Omega \\ 0<r<\operatorname{diam} \Omega}} r^{q_{+}\left(k-n / p_{-}\right)} \int_{B(x, r)}(v(y))^{q(y)} d y<\infty,
$$

then there exists a positive constant $c$ such that

$$
\|u\|_{L_{v}^{q(\cdot)}(\Omega)} \leq c\left\|D^{k} u\right\|_{L^{p(\cdot)}(\Omega)}
$$


for all real-valued functions $u$ in $\Omega$ whose continuation by zero outside $\Omega$ has weak derivatives up to the order $k$ over $R^{n}$.

Proof. Following [3] it can be shown that $D^{k} u \in L^{1}\left(R^{n}\right)$, and therefore (see [15], Theorem 1.1. 10/2) a constant $c_{1}$, depending only on $n$ and $k$, exists such that

$$
|u(x)| \leq c_{1} \int_{\Omega} \frac{\left|D^{k} u(y)\right|}{|x-y|^{n-k}} d y .
$$

Then using condition (3.5) and Theorem II we conclude that (3.6) is valid.

The proof of the next statement is based on the ideas used in [3].

Proposition 3.4. Let $n \geq 2$ and let $1<p_{-}<q_{+}<\infty$. Suppose that the functions $p$ and $q$ satisfy the condition of Theorem II and that $k$ is any positive integer smaller than $n / p_{-}$. If condition (3.5) holds and $\Omega$ is convex, then there exists a positive constant $c$ depending only on $n, k$ and $\Omega$ such that

$$
\inf _{P \in \mathcal{P}_{k-1}}\|u-P\|_{L_{v}^{q(\cdot)}(\Omega)} \leq c\left\|D^{k} u\right\|_{L^{p(\cdot)}(\Omega)}
$$

for all real-valued functions $u$ in $\Omega$ having weak derivatives up to the order $k$ in $\Omega$, where $\mathcal{P}_{m}$ denotes the space of polynomials of order less than or equal to $m$. If $k=1$ inequality (3.7) holds in particular with $P=\frac{1}{\Omega} \int_{\Omega} u(x) d x$.

Proof. It is known (see [15], Theorem 1.1.10/1 and [3]) there exists a positive constant $c_{2}$ depending only on $n, k$ and $\Omega$ and a polynomial $P \in \mathcal{P}_{k-1}$ depending on $n$ such that

$$
|u(x)-P(x)| \leq c_{2} \int_{\Omega} \frac{\left|D^{k} u(y)\right|}{|x-y|^{n-k}} d y
$$

for all $x \in \Omega$ and $u, D^{k} u \in L^{1}(\Omega)$. Theorem II and condition (3.5) completes the proof.

\section{Potentials on Fractal Sets}

Let $\Gamma$ be a subset of $R^{n}$ which is an $s$-set $(0<s \leq n)$ in the sense that there is a Borel measure $\mu$ in $R^{n}$ such that

(i) $\operatorname{supp} \mu=\Gamma$;

(ii) there are positive constants $c_{1}$ and $c_{2}$ such that for all $x \in \Gamma$ and $r \in(0,1)$,

$$
c_{1} r^{s} \leq \mu(B(x, r) \cap \Gamma) \leq c_{2} r^{s} .
$$


It is known that (see [21]) $\mu$ is equivalent to the restriction of Hausdorff $s-$ measure $H_{s}$; we shall thus identify $\mu$ with $\left.H_{s}\right|_{\Gamma}$. Given $x \in \Gamma$, put $\Gamma(x, r)=B(x, r) \cap \Gamma$. Let us indicate some examples of $S H T$ for which the condition (4.1) is satisfied.

Let $\Gamma \subset \mathbb{C}$ be a connected rectifiable curve and let $\nu$ be arc-length measure on $\Gamma$. By definition, $\Gamma$ is regular if

$$
\nu(\Gamma \cap B(z, r)) \leq c r
$$

for every $z \in \mathbb{C}$ and $r>0$.

For $r$ smaller than half the diameter of $\Gamma$, the reverse inequality

$$
\nu(\Gamma \cap B(x, r)) \geq r
$$

holds for all $z \in \Gamma$. Equipped with $\nu$ and the Euclidean metric, the regular curve becomes an $S H T$.

Now let

$$
T^{\alpha(t)} f(t)=\int_{\Gamma} \frac{f(\tau)}{|t-\tau|^{1-\alpha(t)}} d \tau
$$

be an integral with weak variable singularities.

The Cantor set in $R^{n}$ is an $s$ - set, where

$$
s=\frac{\log \left(3^{n}-1\right)}{\log 3} .
$$

Consider the potential-type integral transform on a bounded Cantor set $F$ :

$$
J^{\alpha(x)} f(x)=\int_{F} \frac{f(y)}{|x-y|^{s-\alpha(x)}} d H_{s}, \quad 0<\alpha(x)<s .
$$

Then from the previous results we can derive trace inequalities for the operators $T^{\alpha(\cdot)}$ and $J^{\alpha(\cdot)}$. In some cases the statements have the form of criteria.

To illustrate this we present these results for the case of $J^{\alpha(\cdot)}$.

Proposition 4.1. Let $1<\gamma<\lambda<\infty, \alpha \in W-\operatorname{Lip}(F)$ and $\sup \alpha(x)<s / \gamma$. Then the operator $J^{\alpha(\cdot)}$ acts boundedly from $L^{\gamma}(F)$ into $x \in F$

$L_{\rho}^{\lambda}(F)$ if and only if

$$
\sup _{\substack{x \in F \\ 0<r<\operatorname{diam} F}} r^{\lambda(\alpha(x)-s / \gamma)} \int_{\Gamma(x, r)} \rho^{\lambda}(y) d H_{s}(y)<\infty .
$$


Proposition 4.2. Let $p(\cdot)$ and $q(\cdot)$ be measurable functions on $F$ with $1<p_{-}<q_{+}<\infty$, let $\alpha \in W-\operatorname{Lip}(F)$ and $\sup _{x \in F} \alpha(x)<s / p_{-}$and suppose $v$ is a weight. Then the condition

$$
\sup _{\substack{x \in F \\ 0<r<\operatorname{diam} F}} r^{q_{+}\left(\alpha(x)-s / p_{-}\right)} \int_{\Gamma(x, r)}(v(y))^{q(y)} d H_{s}(y)<\infty
$$

implies the boundedness of $J^{\alpha(\cdot)}$ from $L^{p(\cdot)}(\Gamma)$ to $L_{v}^{q(\cdot)}(\Gamma)$.

Proposition 4.3. Let $p(\cdot)$ and $q(\cdot)$ be as in the previous proposition. Suppose that $\alpha \in W-\operatorname{Lip}(F)$ and

$$
s\left(1 / p_{-}-1 / q_{+}\right) \leq \inf _{x \in F} \alpha(x) \leq \sup _{x \in F} \alpha(x)<s / p_{-} .
$$

Then $J^{\alpha(\cdot)}$ acts boundedly from $L^{p(\cdot)}(F)$ into $L^{q(x)}(F)$.

Acknowledgements. We would like to express our gratitude to the referee for his helpful suggestions and remarks.

\section{References}

[1] D. R. Adams, A trace inequality for generalized potentials, Studia Math., 48 (1973), 99-105.

[2] D. R. Adams and L. I. Hedberg, Function spaces and potential theory, Springer-Verlag, Berlin, 1996.

[3] A. Cianchi and D. E. Edmunds, On fractional integration in weighted Lorentz spaces, Quarterly J. Math. Oxford Ser., (2) 48 (1997), 439-451.

[4] L. Diening, Riesz potential and Sobolev embeddings on generalized Lebesgue and Sobolev spaces $L^{p(\cdot)}$ and $W^{k, p(\cdot)}$, Preprint Mathematische Fakultät, Albert-Ludwigs-Universiẗ̈ Freiburg, 22/2002, 15.07.2002, 113, 2002.

[5] L. Diening and M. Ružička, Calderón-Zygmund operators on generalized Lebesgue spaces $L^{p(x)}$ and problems related to fluid dynamics, Preprint Mathematische Fakultät, Albert-Ludwigs-Universiẗ̈ Freiburg, 21, 2002, 1-20.

[6] D. E. Edmunds, V. Kokilashvili and A. Meskhi, Bounded and compact integral operators, Kluwer Academic Publishers, Dordrecht, Boston, London, 2002.

[7] D. E. Edmunds, I. Lang and A. Nekvinda, On $L^{p(x)}$ norms, R. Soc. Lond. Proc. Ser A. Math. Phys. Eng. Sci., 455 (1999), 219-225. 
[8] D. E. Edmunds and A. Meskhi, Potential type operators in $L^{p(x)}$ spaces, Z. Anal. Anwendungen, 21(2002), 3, 681-690.

[9] D. E. Edmunds and J. Rákosník, Density of smooth functions in $W^{k, p(x)}(\Omega)$, Proc. R. Soc. London, 437 (229-236), 1992.

[10] I. Genebashvili, A. Gogatishvili, V. Kokilashvili and M. Krbec, Weighted theory for integral transforms on spaces of homogeneous type, Pitman Monographs and Survays in Pure and Applied Mathematics, 92, Longman, Harlow, 1998.

[11] H. Hudzik, On generalized Orlicz-Sobolev spaces, Funct. Approx., 4 (1997), 37-51.

[12] V. Kokilashvili and S. Samko, Maximal and fractional operators in weighted $L^{p(x)}$-spaces, Rev. Mat. Iberoamericana (accepted for publication).

[13] V. Kokilashvili and S. Samko, On Sobolev theorem for Riesz potentials in Lebesgue spaces with variable exponent (to appear).

[14] O. Kováčik and J. Rákosník, On spaces $L^{p(x)}$ and $W^{k, p(x)}$, Czechoslovak Math. J., 41 (116) (1991), No. 4, 592-618.

[15] V. G. Maz'ya, Sobolev spaces, Springer, Berlin, 1985.

[16] M. Ružiĉka, Electrorheological fluids : modeling and mathematical theory, Lecture Notes in Mathematics, 1748, Springer, Berlin, 2000.

[17] S. G. Samko, Convolution and potential type operators in $L^{p(x)}\left(R^{n}\right)$, Integral Transform. Spec. Funct., 7 (3-4) (1998), 261-284.

[18] S. Samko, Density $C_{0}^{\infty}\left(R^{n}\right)$ in the generalized Sobolev spaces $W^{m, p(x)}\left(R^{n}\right)$ (Russian) Dokl. Akad. Nauk., 369 (4) (1999), 451-454.

[19] E. T. Sawyer, R. L. Wheeden and S. Zhao, Weighted norm inequalities for operators of potential type and fractional maximal functions, Potential Anal., 5 (1996), 523-580.

[20] J. O. Strömberg and A. Torchinsky, Weighted Hardy spaces, Lecture Notes in Math., 1381, Springer Verlag, Berlin, 1989.

[21] H. Triebel, Fractals and spectra related to Fourier analysis and function spaces, Birkhäuser, Basel, 1997.

[22] V. V. Zhikov, Averaging of function of calculus of variations and elasticity theory, Math. USSR Izv., 29 (1987), 33-66. 
David E. Edmunds

University of Sussex

Centre for Math. Anal. \& Appl.

Brighton BN1 9QH, Sussex

United Kingdom

(E-mail : d.e.edmunds@sussex.ac.uk)

Vakhtang Kokilashvili and Alexander Meskhi

A. Razmadze Mathematical Institute

Georgian Academy of Sciences

1, M. Aleksidze St, 380093 Tbilisi

Georgia

(E-mails : kokil@rmi.acnet.ge ; meskhi@rmi.acnet.ge)

(Received: May 2003) 


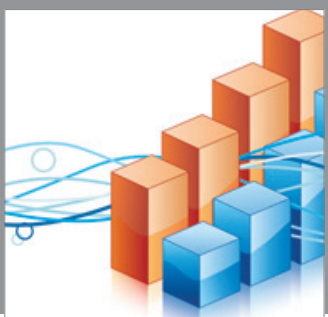

Advances in

Operations Research

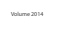

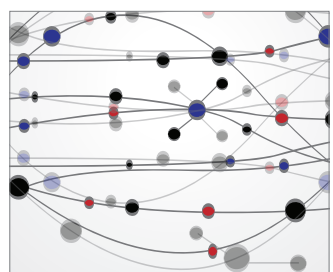

\section{The Scientific} World Journal
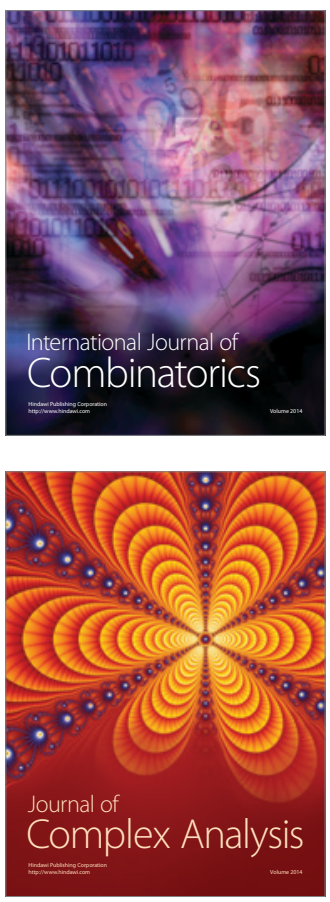

International Journal of

Mathematics and

Mathematical

Sciences
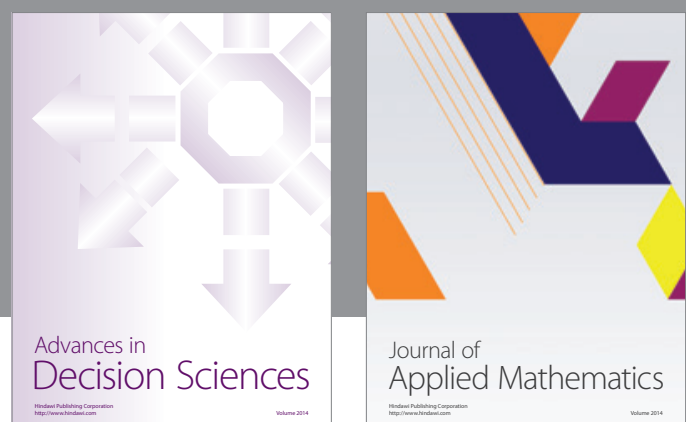

Journal of

Applied Mathematics
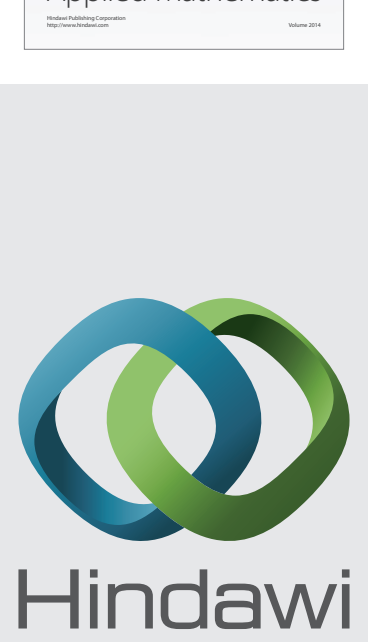

Submit your manuscripts at http://www.hindawi.com
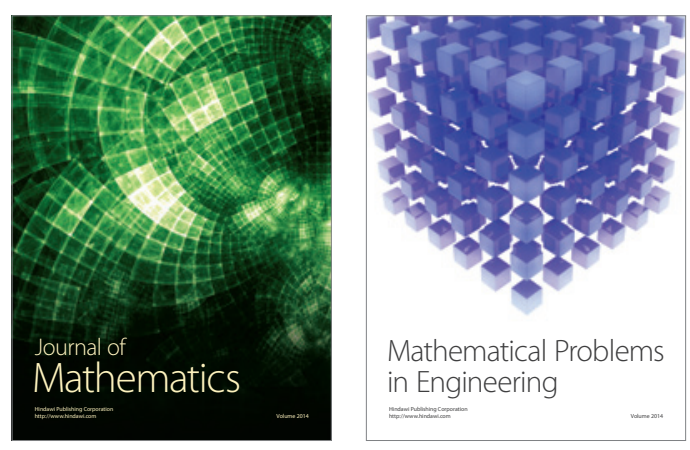

Mathematical Problems in Engineering
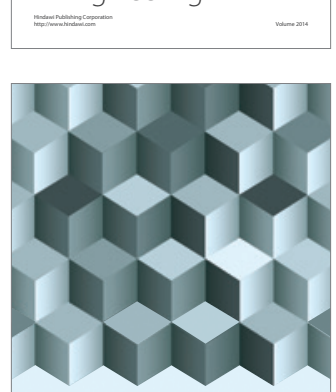

Journal of

Function Spaces
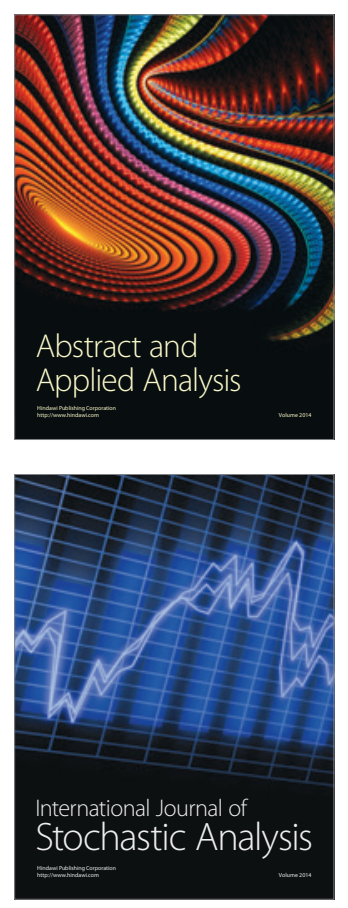

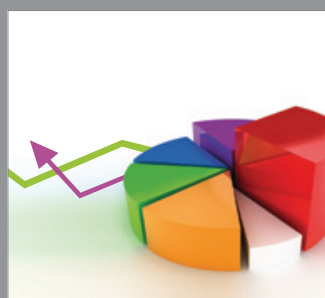

ournal of

Probability and Statistics

Promensencen
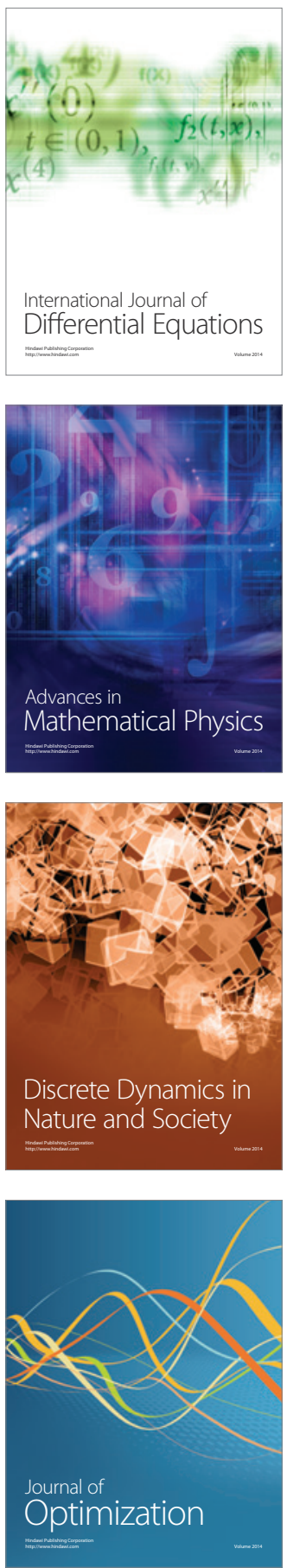